\title{
Sleep in Ionely heroin-dependent patients receiving methadone maintenance treatment: longer sleep latency, shorter sleep duration, lower sleep efficiency, and poorer sleep quality
}

\author{
Hong-Jie Li ${ }^{1, *}$, Bao-Liang Zhong ${ }^{1, *}$, Yan-Min Xu ${ }^{1}$, Jun-Hong Zhu ${ }^{1}$ and Jin Lu ${ }^{2}$ \\ ${ }^{1}$ Affiliated Wuhan Mental Health Center (The Ninth Clinical School), Tongji Medical College of Huazhong University of Science \\ \& Technology, Wuhan, Hubei, China \\ 2 Department of Psychiatry, The First Affiliated Hospital of Kunming Medical University, Kunming, Yunnan Province, China \\ * These authors have contributed equally to this work
}

Correspondence to: Jin Lu, email: jinlu2000@163.com

Keywords: sleep, loneliness, heroin dependence, methadone

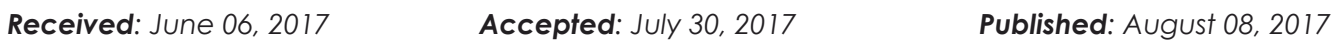

Copyright: Li et al. This is an open-access article distributed under the terms of the Creative Commons Attribution License 3.0 (CC BY 3.0), which permits unrestricted use, distribution, and reproduction in any medium, provided the original author and source are credited.

\section{ABSTRACT}

Given the socially isolated status of Chinese heroin-dependent patients (HDPs) and the significant association between loneliness and sleep problem in the general population, the impact of loneliness on sleep of HDPs is potentially substantial. The study aimed to test whether loneliness is associated with poor sleep in terms of quantity and quality in a consecutive sample of Chinese HDPs receiving methadone maintenance treatment (MMT). The study participants were 603 HDPs of three MMT clinics in Wuhan, China. Data on socio-demographic and clinical characteristics were collected by a standardized self-administered questionnaire. Sleep outcomes included sleep latency, sleep duration, sleep efficiency, and sleep quality. We measured depressive symptoms, loneliness, and sleep quality by using Zung's Self-rating Depression Scale, the single-item self-report of loneliness, and the Pittsburgh Sleep Quality Index, respectively. Multiple linear regression was used to examine whether loneliness is independently associated with sleep measures. After controlling for the confounding effects of potential socio-demographic and clinical variables, loneliness was significantly associated with longer sleep latency, shorter sleep duration, lower sleep efficiency, and poorer sleep quality. Loneliness may exacerbate sleep disturbance in Chinese HDPs of MMT clinics. Psychosocial interventions aimed at reducing loneliness in MMT clinics would improve the sleep of HDPs.

\section{INTRODUCTION}

Sleep disturbance is highly prevalent among heroin-dependent patients (HDPs) [1-3]. A meta-analysis based on 13 studies with 1857 patients reported a 78.5\% prevalence of sleep problem in HDPs [4]. Evidence from empirical studies has shown that, in HDPs, sleep disorder is significantly associated with addiction relapse, multiple drug use, and poorer quality of life [4-7]. Therefore, sleep problem is of significant clinical interest in addiction treatment practice. To date, there have been many studies investigating factors that are significantly associated with HDPs' sleep problem [2-5, 8-11], however, nearly all these studies focused on unmodifiable factors of sleep disturbance among HDPs, such as unemployment, long length of heroin use, and a history of injecting heroin. By contrast, very few research has studied factors that can be treated or changed.

Loneliness is defined as "the unpleasant experience that occurs when a person's network of social relations is deficient in some important way, either quantitatively or qualitatively" $[12,13]$. In China, due to the strong social stigma towards persons with heroin addiction [14], HDPs often experience social isolation and loneliness [15]. Accumulating evidence from population-based studies have shown that loneliness is a significant risk factor for a variety of physical and mental health problems, including depression, dementia, increased blood pressure 
and sleep disturbance [16-18]. Recent studies further suggest that diminished sleep is a potentially pathway by which loneliness adversely affect health [18-20]. Because loneliness and sleep problem are preventable or treatable [16], a better understanding on how loneliness affects sleep would facilitate the development of measures to reduce the many harmful effects of loneliness.

To the best our knowledge, no studies have investigated the association between loneliness and sleep problem of HDPs. Importantly, because psychosocial services are not routinely provided in Chinese addiction treatment practice [21], untreated loneliness would have a greater negative effect on the sleep of Chinese HDPs if it is true that loneliness is significantly associated with sleep disturbance. This study set out to examine the impact of loneliness on sleep patterns in a sample of HDPs receiving methadone maintenance treatment (MMT). Because previous studies showed that lonely people spent much more time in bed but much less time sleeping and loneliness impaired daytime functioning [20, 22, 23], the hypothesis of this study was that loneliness would be associated with longer sleep latency, shorter sleep duration, lower sleep efficiency, and poorer sleep quality.

\section{RESULTS}

In total, 603 HDPs completed the survey. Of the 603 participants, $412(68.3 \%)$ were males, and the average age was 38.1 years (standard deviation $[\mathrm{SD}]=7.0$, range $=21-59$ ). Their mean sleep latency, duration, efficiency, and quality score (PSQI) were $35.7 \mathrm{~min}$. $(\mathrm{SD}=34.2), 9.0$ hours $(\mathrm{SD}=2.3), 87.5 \%(\mathrm{SD}=15.3)$, and $6.6(\mathrm{SD}=3.8)$, respectively. Numbers (percentage) of patients who felt lonely "never", "seldom", "sometimes", "often", and "always" were 7 (1.2\%), 259 (43.0\%), 160 $(26.5 \%), 141(23.4 \%)$, and $36(6.0 \%)$, respectively. A total of 337 patients sometimes or more frequently felt lonely; the prevalence of loneliness was $55.9 \%$. Detailed socio-demographic and clinical characteristics of these respondents are displayed in Table 1.

As shown in Table 1, compared to not lonely patients, lonely patients had significantly longer sleep latency $(\mathrm{z}=3.111, \mathrm{P}=0.002)$, shorter sleep duration $(\mathrm{t}=2.807, \mathrm{P}=0.005)$, lower sleep efficiency $(\mathrm{t}=2.548$, $\mathrm{P}=0.011)$, and higher PSQI score $(\mathrm{t}=2.752, \mathrm{P}=0.006)$. Table 1 also shows that these sleep measures significantly differed between some subgroups according to sociodemographic and clinical characteristics, indicating that socio-demographic and clinical variables may bias the association between loneliness and sleep measures. After controlling for potential socio-demographic and clinical confounders, results of the multiple linear regression analyses (Table 1) reveal that loneliness remained significantly associated with longer sleep latency (unstandardized coefficient $[$ Beta] $=10.195, \mathrm{P}=0.006$ ), shorter sleep duration (Beta=-0.611, $\mathrm{P}=0.009$ ), lower sleep efficiency $($ Beta $=-3.402, \mathrm{P}=0.037)$, and poorer sleep quality (Beta $=0.907, \mathrm{P}=0.008)$, respectively.

\section{DISCUSSION}

In this study, the estimated prevalence of loneliness was $55.9 \%$ among Chinese HDPs of MMT clinics. In comparison with studies using similar definitions of loneliness, this prevalence is much higher than that reported in Chinese rural-to-urban migrant workers $(18.3 \%)$ and older adults (33\%) [13, 17]. The high prevalence of loneliness in HDPs found in this study is concordant with the significantly higher level of loneliness experienced by young MDMA users and individuals with substance use disorder compared with healthy controls $[24,25]$.

Previous studies have found that the sleep of methadone-maintained HDPs is characterized by longer latency, shorter duration, lower efficiency, more awakenings, shorter slow wave sleep, and poorer sleep quality $[10,26]$. Our study revealed that, in methadonemaintained HDPs, lonely individuals relative to not lonely individuals had longer sleep latency, shorter sleep duration, lower sleep efficiency, and poorer sleep quality, and the influence of loneliness on sleep was independent of socio-demographic and clinical covariates. These findings suggest that lonely HDPs have more difficulties in initiating and maintaining sleep, and the two together result in poor sleep quality.

Endogenous opioid peptides play an important role in the onset and maintenance of sleep and sleep/wake transition $[26,27]$. Because opioid receptors can also be activated by exogenous opiates [26], heroin suppresses the endogenous opioid systems in the brain. Due the feedback inhibition of endogenous opioid system caused by longterm use of heroin, HDPs may exhibit difficulties in sleep initiation and maintenance such as longer latency and shorter duration.

Existing studies on the impact of loneliness on sleep of the general population found that loneliness is significantly associated with daytime dysfunction, sleep fragmentation, and poor sleep quality, but not sleep duration [18-20]. It seems that loneliness does affect various measures of sleep other than the amount of sleep. Our findings are not fully consistent with results from these literature. Differences in subjects (older adults vs. HDPs) and measures of sleep (i.e., subjective vs. objective) may explain such discrepancy. However, it is also possible that loneliness has a more profound negative effect on sleep of HDPs, and thus leads to impairments in both quantity and quality of sleep. There are two possible reasons for the loneliness-sleep link. First, there is evidence that feelings of loneliness are associated with an increase in circulating cortisol and a high level of cortisol triggers the stress response [19, 28, 29], which in turn keeps lonely individuals awake at night. Second, 
Table 1: Latency, duration, efficiency and quality of sleep according to socio-demographic and clinical characteristics of HDPs receiving MMT

\begin{tabular}{|c|c|c|c|c|c|c|c|c|c|}
\hline Characteristics & $n$ & $\begin{array}{l}\text { Latency } \\
(\mathrm{min} .)^{\#}\end{array}$ & $\begin{array}{l}\text { Unstandardized } \\
\text { Beta }\end{array}$ & $\begin{array}{l}\text { Duration } \\
\text { (hours) }^{\$}\end{array}$ & $\begin{array}{l}\text { Unstandardized } \\
\text { Beta }\end{array}$ & Efficiency $(\%)^{\mathrm{s}}$ & $\begin{array}{l}\text { Unstandardized } \\
\text { Beta }\end{array}$ & Quality & $\begin{array}{l}\text { Unstandardized } \\
\text { Beta }\end{array}$ \\
\hline \multicolumn{10}{|l|}{ Gender } \\
\hline Male & 412 & $38.6 \pm 38.3^{* *}$ & 6.737 & $8.7 \pm 2.1 * * *$ & $-1.065^{* * *}$ & $87.0 \pm 15.2$ & -1.803 & $6.7 \pm 3.6$ & -0.283 \\
\hline Female & 191 & $29.4 \pm 22.2$ & Reference & $9.6 \pm 2.7$ & Reference & $88.5 \pm 15.5$ & Reference & $8.7 \pm 2.1$ & Reference \\
\hline \multicolumn{10}{|l|}{ Age (years) } \\
\hline $20-39$ & 327 & $36.6 \pm 28.5^{* *}$ & 3.722 & $9.1 \pm 2.3$ & $0.623 * *$ & $87.1 \pm 14.8$ & -2.271 & $6.6 \pm 3.7$ & -0.216 \\
\hline $40-59$ & 276 & $34.5 \pm 40.0$ & Reference & $8.8 \pm 2.4$ & Reference & $87.9 \pm 16.0$ & Reference & $6.7 \pm 4.0$ & Reference \\
\hline \multicolumn{10}{|l|}{ Education years } \\
\hline$<9$ & 140 & $34.9 \pm 22.7$ & -2.437 & $9.3 \pm 2.4$ & $0.525^{*}$ & $88.1 \pm 14.8$ & 1.135 & $6.6 \pm 3.8$ & 0.226 \\
\hline$\geq 9$ & 463 & $35.0 \pm 36.1$ & Reference & $9.0 \pm 2.3$ & Reference & $88.1 \pm 14.4$ & Reference & $6.6 \pm 3.9$ & Reference \\
\hline \multicolumn{10}{|l|}{ Marital status } \\
\hline Married & 295 & $34.4 \pm 38.4^{*}$ & $-1.249 * *$ & $9.1 \pm 2.5$ & 0.393 & $88.7 \pm 14.0$ & -0.558 & $6.4 \pm 3.7$ & -0.084 \\
\hline Non-married\& & 308 & $35.6 \pm 28.1$ & Reference & $9.0 \pm 2.2$ & Reference & $87.5 \pm 15.0$ & Reference & $6.8 \pm 4.0$ & Reference \\
\hline \multicolumn{10}{|l|}{ Employment } \\
\hline Yes & 271 & $34.3 \pm 38.1 * *$ & $-1.432 *$ & $9.4 \pm 2.5^{* *}$ & $0.547 *$ & $90.8 \pm 12.2 * * *$ & $5.355^{* * *}$ & $5.9 \pm 3.5 * * *$ & $-1.042 * *$ \\
\hline No & 332 & $36.7 \pm 28.7$ & Reference & $8.7 \pm 2.2$ & Reference & $85.6 \pm 16.2$ & Reference & $7.4 \pm 4.0$ & Reference \\
\hline \multicolumn{10}{|c|}{$\begin{array}{l}\text { Self-rated economic } \\
\text { status\& }\end{array}$} \\
\hline Good & 149 & $34.0 \pm 42.4$ & 1.023 & $8.9 \pm 2.1$ & $-0.412^{*}$ & $91.2 \pm 13.3^{*}$ & 1.022 & $5.8 \pm 3.4^{* *}$ & -1.386 \\
\hline Fair & 314 & $36.8 \pm 32.4$ & 1.976 & $9.1 \pm 2.4$ & -0.525 & $87.0 \pm 14.5$ & 0.542 & $6.8 \pm 3.7$ & -2.579 \\
\hline Poor & 140 & $33.5 \pm 24.7$ & Reference & $9.2 \pm 2.4$ & Reference & $86.9 \pm 15.4$ & Reference & $7.1 \pm 4.6$ & Reference \\
\hline \multicolumn{10}{|l|}{$\begin{array}{l}\text { Route of heroin } \\
\text { administration }\end{array}$} \\
\hline Smoking & 96 & $25.6 \pm 22.3^{* * *}$ & $-9.849^{*}$ & $9.9 \pm 2.4 * * *$ & $1.004 * * *$ & $91.7 \pm 13.7^{* *}$ & $2.783^{*}$ & $5.6 \pm 3.7 * * *$ & $-0.494 * *$ \\
\hline Injecting & 507 & $36.8 \pm 35.0$ & Reference & $8.9 \pm 2.3$ & Reference & $87.4 \pm 14.6$ & Reference & $6.8 \pm 3.8$ & Reference \\
\hline \multicolumn{10}{|c|}{$\begin{array}{l}\text { Duration of heroin } \\
\text { use (years) }\end{array}$} \\
\hline$<10$ & 230 & $32.7 \pm 27.0^{* *}$ & $-2.497^{*}$ & $9.4 \pm 2.3^{* * *}$ & 0.347 & $89.8 \pm 12.7^{* * *}$ & $4.481 * *$ & $6.0 \pm 3.5^{* * *}$ & $-1.051 * *$ \\
\hline$\geq 10$ & 373 & $38.5 \pm 41.5$ & Reference & $8.5 \pm 2.3$ & Reference & $85.3 \pm 16.6$ & Reference & $7.5 \pm 4.2$ & Reference \\
\hline \multicolumn{10}{|c|}{$\begin{array}{l}\text { Duration of MMT } \\
\text { (months) }\end{array}$} \\
\hline$<24$ & 240 & $30.0 \pm 19.5$ & -2.211 & $9.2 \pm 2.2$ & 0.168 & $89.2 \pm 13.8$ & 1.668 & $5.9 \pm 3.4 * * *$ & $-0.836^{* *}$ \\
\hline$\geq 24$ & 363 & $38.1 \pm 39.9$ & Reference & $9.0 \pm 2.4$ & Reference & $87.2 \pm 14.9$ & Reference & $7.1 \pm 4.0$ & Reference \\
\hline \multicolumn{10}{|c|}{$\begin{array}{l}\text { Methadone dosage } \\
\text { (mg/day) }\end{array}$} \\
\hline$<70$ & 273 & $34.2 \pm 28.3$ & -0.187 & $9.0 \pm 2.1$ & -0.009 & $88.6 \pm 13.4$ & 0.465 & $6.2 \pm 3.5$ & 0.351 \\
\hline$\geq 70$ & 330 & $35.6 \pm 37.2$ & Reference & $9.1 \pm 2.5$ & Reference & $87.7 \pm 15.3$ & Reference & $6.9 \pm 4.1$ & Reference \\
\hline Depression & & & & & & & & & \\
\hline
\end{tabular}




\begin{tabular}{|l|l|l|l|l|l|l|l|l|l|}
\hline Yes & 204 & $44.1 \pm 37.3^{* * *}$ & $14.612^{* * *}$ & $9.1 \pm 2.3$ & 0.177 & $86.5 \pm 15.0^{*}$ & $-0.641^{*}$ & $8.2 \pm 4.0^{* * *}$ & $2.391^{* * *}$ \\
\hline No & 399 & $29.0 \pm 30.0$ & Reference & $9.0 \pm 2.4$ & Reference & $89.4 \pm 13.5$ & Reference & $5.3 \pm 3.1$ & Reference \\
\hline Loneliness & & & & & & & & & \\
\hline Yes & 337 & $37.4 \pm 31.9^{* *}$ & $10.195^{* *}$ & $8.8 \pm 2.3^{* *}$ & $-0.611^{* *}$ & $86.3 \pm 14.7^{* *}$ & $-3.402^{*}$ & $7.4 \pm 3.9^{* * *}$ & $0.907^{* *}$ \\
\hline No & 266 & $32.7 \pm 35.4$ & Reference & $9.4 \pm 2.3$ & Reference & $90.2 \pm 14.0$ & Reference & $5.6 \pm 3.5$ & Reference \\
\hline
\end{tabular}

\#Mann-Whitney U test.

${ }^{s} t$-test.

\& one-way analysis of variance.

\&"Non-married" included never-married, remarried, cohabitating, separated/divorced, and widowed.

$* P<0.05, * * P<0.01, * * * P<0.001$.

the association between loneliness and poor sleep may also be explained by the heightened vigilance in lonely individuals, because people need a safe social surround to sleep soundly but lonely individuals are driven by increased vigilance for threat [19].

The present study has several limitations. Due to the cross-sectional nature of the study, we can not determine whether loneliness precedes sleep problem and infer the causal relationship between loneliness and sleep problem. Second, comparing the nocturnal cortisol levels of lonely and not lonely HDPs might help explain the loneliness-sleep link, but we did not measure this potentially useful biomarker due to deficiencies in the study design. Third, some other related factors of sleep problem (negative life events, lack of social support, etc.) were not evaluated in the study so our analysis is not able to exclude the confounding effects of these factors, which might influence the loneliness-sleep association. Finally, measures of sleep used in this study are subjective in nature, which are different from those collected objectively such as polysomnography. More studies are warranted to explore the impact of loneliness on objective measures of sleep.

In summary, there is a significant association between loneliness and poor sleep in terms of quality and quantity, including longer sleep latency, shorter sleep duration, lower sleep efficiency, and poorer sleep quality, indicating that loneliness may exacerbates sleep disturbance of HDPs. Given the high prevalence of loneliness in HDPs and the unavailability of psychosocial services in Chinese MMT clinics, providing psychosocial services to reduce or prevent loneliness might be an effective way to reduce sleep problem of methadonemaintained HDPs.

\section{MATERIALS AND METHODS}

\section{Subjects}

This cross-sectional study was conducted in three MMT clinics in Wuhan, China, between December 2009 and March 2010. A consecutive sample of adult patients who met DSM-IV criteria for lifetime heroin dependence and were taking methadone for drug rehabilitation in these MMT clinics, was invited to participate in this study. Patients with psychotic symptoms, serious physical illnesses, alcohol dependence, or brain organic mental disorders, were excluded.

The study protocol was approved by the Ethics Committee of Wuhan Mental Health Center. All participants provided written informed consent before the fieldwork of the survey.

\section{Instruments and procedures}

This study used a self-administered questionnaire to collect data. Sleep quantity and quality in the past month were main outcomes of this study. The former included total hours of actual sleep at night (sleep duration), minutes taken to fall asleep each night (sleep latency), and the percentage of total actual sleep time out of the total time in bed (sleep efficiency). Sleep quality was measured with the Pittsburgh Sleep Quality Index (PSQI) [30], which is a widely used self-rated scale that assesses sleep quality and disturbances over the previous month. It has 19 items and each item is rated on a four-point integer scale (0-3). The total PSQI score is calculated by totaling the 19 item scores, with higher scores denoting a poorer sleep quality. Studies have proved the good reliability and validity of Chinese PSQI in both clinical and non-clinical settings [31-33].

Socio-demographic variables collected in the questionnaire were gender, age, education years, marital status, employment status, and self-rated economic status (good, fair, poor). Clinical characteristics collected included usual route of heroin administration (smoking, injecting), duration of heroin use, duration of MMT, methadone dosage, and depression. Depression was assessed with Zung's Self-rating Depression Scale (SDS) [34]. The Chinese SDS is a 20 -item self-rating scale to evaluate the severity of depressive symptoms and each item is scored on a 4-point scale ( $1=a$ little of the time, $2=$ some of the time, $3=$ good part of the time, $4=$ most of the time) [35]. Total SDS score varies between 20 and 
80 and a cut-off score of 40 or greater is used to indicate clinically significant depression [34].

Loneliness was assessed by using a single question: "How often you feels lonely?". The responses for this question were: $1=$ always, $2=$ often, $3=$ sometimes, $4=$ seldom, $5=$ never. This single-item measure of loneliness was widely used in prior studies [13, 36-38]. In line with prior studies [16, 36, 37], patients were classified as having loneliness if they felt lonely at least sometimes.

\section{Statistical analysis}

Participants were divided into different subgroups (i.e,. male vs. female) according to their sociodemographic and clinical characteristics. Sleep measures of these subgroups were described and compared by t-test, one-way analysis of variance, or Mann-Whitney $\mathrm{U}$ test, as appropriate. The effect of loneliness on sleep was assessed with multiple linear regression that entered a sleep measure as the outcome variable, loneliness as the predictor, and socio-demographic and clinical covariates at once to adjust for the potential confounding effects of these socio-demographic and clinical variables. The statistical significance level was set at $\mathrm{p}<0.05$ (twosided). SPSS software version 15.0 package was used for analyses.

\section{ACKNOWLEDGMENTS}

The authors thank all the patients involved in this study for their cooperation and support.

\section{CONFLICTS OF INTEREST}

The authors declare no conflicts of interest.

\section{GRANT SUPPORT}

This study was supported by Wuhan Health and Family Planning Commission [WX17Q30, YM Xu, PI; WG16A02, BL Zhong, PI] and Health and Family Planning Commission of Yunnan Province [2016NS027, $\mathrm{J} \mathrm{Lu}, \mathrm{PI}]$.

\section{REFERENCES}

1. Khazaie H, Najafi F, Ghadami MR, Azami A, Nasouri M, Tahmasian M, Khaledi-Paveh B. Sleep Disorders in Methadone Maintenance Treatment Volunteers and Opiumdependent Patients. Addict \& health. 2016; 8:84-89.

2. Zahari Z, Siong LC, Musa N, Mohd Yasin MA, Choon TS, Mohamad N, Ismail R. Report: Demographic profiles and sleep quality among patients on methadone maintenance therapy (MMT) in Malaysia. Pak J Pharm Sci. 2016; 29:239-246.
3. Tang J, Liao Y, He H, Deng Q, Zhang G, Qi C, Cui H, Jiao B, Yang M, Feng Z, Chen X, Hao W, Liu T. Sleeping problems in Chinese illicit drug dependent subjects. BMC Psychiatry. 2015; 15:28.

4. Xu YM, Zhong BL, Liu TB. The prevalence of sleep problem among heroin addicts: a meta-analysis. Chin J Drug Depend. 2014; 23:369-374.

5. Liao Y, Tang J, Liu T, Chen X, Luo T, Hao W. Sleeping problems among Chinese heroin-dependent individuals. Am J Drug Alcohol Abuse. 2011; 37:179-183.

6. Roth T, Workshop Participants. Does effective management of sleep disorders reduce substance dependence? Drugs. 2009 (Suppl 2); 69:65-75.

7. Zhu JH, Zhong BL. Effect of sleep quality on quality of life of heroin addicts receiving methadone maintenance treatment. Chin J Behav Med \& Brain Sci. 2013; 22:607610.

8. Peles E, Schreiber S, Adelson M. Variables associated with perceived sleep disorders in methadone maintenance treatment (MMT) patients. Drug Alcohol Depend. 2006; 82:103-110.

9. Hsu WY, Chiu NY, Liu JT, Wang CH, Chang TG, Liao YC, Kuo PI. Sleep quality in heroin addicts under methadone maintenance treatment. Acta Neuropsychiatrica. 2012; 24:356-360.

10. Xu YM, Zhong BL, Zhu JH, Liu TB. Sleep characteristics of heroin addicts receiving methadone maintenance treatment. Chin J Drug Depend. 2014; 23:458-463.

11. Chen VC, Ting H, Wu MH, Lin TY, Gossop M. Sleep disturbance and its associations with severity of dependence, depression and quality of life among heroindependent patients: a cross-sectional descriptive study. Subst Abuse Treat Prev Policy. 2017; 12:16.

12. Perlman D, Peplau L. (1981). Toward a social psychology of loneliness. In: Gilmour R and Duck S, eds. Personal Relationships, Volume 3: Personal Relationships in Disorder. (London: Academic Press).

13. Zhong BL, Chen SL, Tu X, Conwell Y. Loneliness and Cognitive Function in Older Adults: Findings From the Chinese Longitudinal Healthy Longevity Survey. J Gerontol B Psychol Sci Soc Sci. 2017; 72:120-128.

14. Lembke A, Zhang N. A qualitative study of treatmentseeking heroin users in contemporary China. Addict Sci Clin Pract. 2015; 10:23.

15. Yang M, Mamy J, Gao P, Xiao S. From Abstinence to Relapse: A Preliminary Qualitative Study of Drug Users in a Compulsory Drug Rehabilitation Center in Changsha, China. PLoS One. 2015; 10:e0130711.

16. Zhong BL, Chen SL, Conwell Y. Effects of Transient Versus Chronic Loneliness on Cognitive Function in Older Adults: Findings From the Chinese Longitudinal Healthy Longevity Survey. Am J Geriatr Psychiatry. 2016; 24:389398.

17. Zhong B, Xu Y, Jin D, Zou X, Liu T. Prevalence and 
correlates of loneliness among Chinese service industry migrant workers: A cross-sectional survey. Medicine. 2016; 95:e3903.

18. Kurina LM, Knutson KL, Hawkley LC, Cacioppo JT, Lauderdale DS, Ober C. Loneliness is associated with sleep fragmentation in a communal society. Sleep. 2011; 34:1519-1526.

19. Matthews T, Danese A, Gregory AM, Caspi A, Moffitt TE, Arseneault L. Sleeping with one eye open: loneliness and sleep quality in young adults. Psychol Med. 2017:1-10.

20. Hawkley LC, Preacher KJ, Cacioppo JT. Loneliness impairs daytime functioning but not sleep duration. Health Psychol. 2010; 29:124-129.

21. Zhong B, Xiang Y, Cao X, Li Y, Zhu J, Chiu HF. Prevalence of antisocial personality disorder among Chinese individuals receiving treatment for heroin dependence: a meta-analysis. Shanghai Arch Psychiatry. 2014; 26:259271.

22. Cacioppo JT, Hawkley LC, Berntson GG, Ernst JM, Gibbs AC, Stickgold R, Hobson JA. Do lonely days invade the nights? Potential social modulation of sleep efficiency. Psychol Sci. 2002; 13:384-387.

23. Hawkley LC, Cacioppo JT. Loneliness matters: a theoretical and empirical review of consequences and mechanisms. Ann Behav Med. 2010; 40:218-227.

24. Hosseinbor M, Yassini Ardekani SM, Bakhshani S, Bakhshani S. Emotional and social loneliness in individuals with and without substance dependence disorder. Int J High Risk Behav Addict. 2014; 3:e22688.

25. Rokach A. Determinants of loneliness of young adult drug users. J Psyhol. 2002; 136:613-630.

26. Xiao L, Tang YL, Smith AK, Xiang YT, Sheng LX, Chi Y, Du WJ, Guo S, Jiang ZN, Zhang GF, Luo XN. Nocturnal sleep architecture disturbances in early methadone treatment patients. Psychiatry Res. 2010; 179:91-95.

27. Kryger MH, Roth T, Dement WC. (2017). Principles and Practice of Sleep Medicine (Sixth Edition). (Philadelphia: Elsevier Saunders).

28. Doane LD, Adam EK. Loneliness and cortisol: momentary, day-to-day, and trait associations. Psychoneuroendocrinology. 2010; 35:430-441.
29. Riemann D, Klein T, Rodenbeck A, Feige B, Horny A, Hummel R, Weske G, Al-Shajlawi A, Voderholzer U. Nocturnal cortisol and melatonin secretion in primary insomnia. Psychiatry Res. 2002; 113:17-27.

30. Buysse DJ, Reynolds CF 3rd, Monk TH, Berman SR, Kupfer DJ. The Pittsburgh Sleep Quality Index: a new instrument for psychiatric practice and research. Psychiatry Res. 1989; 28:193-213.

31. Zheng B, Li M, Wang KL, Lv J. [Analysis of the reliability and validity of the Chinese version of Pittsburgh sleep quality index among medical college students]. [Article in Chinese]. Beijing Da Xue Xue Bao. 2016; 48:424-8.

32. Ho RT, Fong TC. Factor structure of the Chinese version of the Pittsburgh sleep quality index in breast cancer patients. Sleep Med. 2014; 15:565-569.

33. Liu XC, Tang MQ, Hu L, Wang AZ, Wu HX, Zhao GF, Gao CN, Li WS. Reliability and validility of the Pittsburgh sleep quality index. Chin J Psychiatry. 1996; 29:103-107.

34. Wang XD, Wang XL, Ma H. (1999). Handbook of Mental Health Rating Scales. (Beijing: Chinese Mental Health Journal Press).

35. Zhong BL, Liu TB, Chan SS, Jin D, Hu CY, Dai J, Chiu HF. Common mental health problems in rural-to-urban migrant workers in Shenzhen, China: prevalence and risk factors. Epidemiol Psychiatr Sci. 2017:1-10.

36. Dahlberg L, Andersson L, McKee KJ, Lennartsson C. Predictors of loneliness among older women and men in Sweden: A national longitudinal study. Aging Ment Health. 2015; 19:409-417.

37. Routasalo PE, Savikko N, Tilvis RS, Strandberg TE, Pitkala KH. Social contacts and their relationship to loneliness among aged people - a population-based study. Gerontology. 2006; 52:181-187.

38. Victor C, Grenade L, Boldy D. Measuring loneliness in later life: a comparison of differing measures. Rev Clin Gerontol. $2005 ; 15: 63-70$. 